\title{
Statistical Analysis of Factors that Affect the Use of Maternal Health Care Services in Gubure Sub-City: SNNP, Ethiopia
}

\section{Gedif Mulat Alemayehu*}

Biostatistics Department, Institute of Public Health, Wolkite University, Wolkite, Ethiopia

\begin{abstract}
Background: Many mothers suffer from complications of pregnancy and delivery problems. Complications of pregnancy and childbirth are a leading cause of maternal morbidities and mortalities in developing countries. The WHO estimates that over 500,000 women and girls die each year from the complications and mortality of mothers lack of antenatal visit care is high in Gubre city.
\end{abstract}

Objective: The aim of this research is to identify the factors that affect the use of maternal health care service.

Methodology: The study was conducted by using simple random sampling and samples size was selected by lottery method and the total sample size selected was 145 out of 1624 total population. The research was conducted by using primary data and the collected data were analysed by statistical software SPSS version 23 , and binary Logistic regression analysis was used to analyse the data.

Results: Based on this analysis marital status, occupation, immunization, background of maternal health services, existence of adequate health services were the major factors on the use of maternal health care services.

Conclusion: From the findings we conclude that many of women are not the user of services because of there is no adequate health services as well as there is no essential information in the population. Therefore we would like to inform to the Gubre sub city that have authority to distribute maternal health services as they constructs for this area and to provide enough treatment for mother's and for all female's. The researchers recommend that appropriate intervention should be done by the government, and the health concerned body.

Keywords: Maternal health care services; Odds ratio; Postpartum period; Morbidity; Mortality; Postnatal

Abbreviations: WHO: World Health Organization; TBAs: Traditional Birth Assistance; UNPF: United Nation Population Fund; MHC: Maternal Health Care; FMOH: Federal Ministry of Health; NGOs: Nongovernmental Organizations; CSA: Central Statistical Agency; MNPI: Maternal and Neonatal Program Index; EDHS: Ethiopia Demographic Health Survey; ANC: Antenatal Care

\section{Introduction}

Maternal health refers to the health of women during pregnancy, childbirth, and the postpartum period. It encompasses the health care dimensions of family planning, preconception, prenatal, and postnatal care in order to reduce maternal morbidity and mortality [1].

Preconception care can include education, health promotion, screening and other interventions among women of reproductive age to reduce risk factors that might affect future pregnancies. The goal of prenatal care is to detect any potential complications of pregnancy early, to prevent them if possible, and to direct the woman to appropriate specialist medical services as appropriate. Postnatal care issues include recovery from childbirth, concerns about new-born care, nutrition, breastfeeding, and family planning [2].

Almost 50\% of the births in developing countries still take place without a medically skilled attendant to aid the mother, and the ration is even higher in developing nations. Women in Sub-Saharan Africa mainly rely on traditional birth attendants (TBAs), who have little or no formal health care training. In recognition of their role, some counties and non-governmental organizations are making efforts to train TBA sin maternal health topics. In order to improve the chances for better health outcomes among mother and babies [3].

In Ethiopia most maternal health services are poor and lives under the condition. For mother adequate care during pregnancy and delivery is essential, However maternal health care services utilization is extremely low. Data show that antenatal care coverage in Ethiopia is only $27.7 \%$.Delivery and postnatal care services are only $5.3 \%$ and $5.8 \%$, respectively [4].

The low utilization of health care services for mothers causes large number death of women in the childbearing age (15-49 years) which is related with complications associated with pregnancy and child birth.According to the EDHS 2011 report, about 17,000 women of reproductive age die from complications associated with pregnancy and birth per year. The maternal mortality ratios in developing countries estimated at 871 per 1,000,000 live births [5].

\section{Research Questions}

1. What are the Utilization of Maternal Health care Services in gurage zone Gubre sub city?

2. What are the sociodemographic, economic and cultural factors affecting maternal health care service utilization Gubre?

3. What are the determinants of healthcare institutional childbirth delivery in Gubre?

${ }^{*}$ Corresponding author: Alemayehu GM, Biostatistics Department, Institute of Public Health, Wolkite University, Wolkite, Ethiopia, Tel: +251920469764; E-mail: gedifmulat@gmail.com

Received April 24, 2018; Accepted June 07, 2018; Published June 17, 2018

Citation: Alemayehu GM (2018) Statistical Analysis of Factors that Affect the Use of Maternal Health Care Services in Gubure Sub-City: SNNP, Ethiopia. J Biom Biostat 9: 399. doi: 10.4172/2155-6180.1000399

Copyright: (C) 2018 Alemayehu GM. This is an open-access article distributed under the terms of the Creative Commons Attribution License, which permits unrestricted use, distribution, and reproduction in any medium, provided the original author and source are credited. 


\section{Methods}

\section{Study design and sample size}

The study was conducted in Gubire sub city which is located in Gurage Zone southern Region of Ethiopia and the study populations were women of reproductive age (15-49 years) who gave at least one live birth in the five years prior to the survey date and who were the usual residents of the district, the total population $(\mathrm{N})$ were 1624 . A community based cross-sectional study design was employed for quantitative data collection method.

The sample size was determined using the formula of Cochran $n=\frac{z^{2} P q}{d^{2}}$. Thus, taking the prevalence of one of the major parameters in this study, that is, antenatal care utilization, pilot survey was used to know $\mathrm{p}$ and $\mathrm{q}$ based on the pilot survey the sample size was estimated to 145 , therefore $\mathrm{n}=145$.

Mothers for the study were selected from the gubre district health center by identfying theire registry card who have children before the 5 year of the survey conducted. Lottery method was applied to select to select individuals for data collecting purpose after the women selected [6-10].

\section{Variables and source of data}

The outcome variables in this study are antenatal and delivery care services utilization for which the categories are either use (coded as 0 ) or non-use (1) of the services.

The independent variables were categorized into demographic which includes age, Marital, Education level status, Level of income, Number of children, Occupation of women, Religion $0=$ orthodox, Nutritional status, Use of immunization, Health services, Back ground information of health services.

The independent variable for this study were selected from the prior studies or literatures, we took some explanatory variables from Ethiopian demographic and health survey (EDHS), and we also add some independent variables from the opinion of garage zone health office experts. The data was entered using EPI INFO and finally was exported to SPSS version 23 for analysis [11-14]. Frequencies and summary statistics such as means, standard deviations, percentages, and ranges were computed to describe the study population in relation to relevant variables. The association and significance between the dependent and independent variables were measured using binary logistic regression analysis.

The questionnaire was adapted from Ethiopian Demographic and Health Survey English version. It was further developed after reviewing of relevant literatures that address the objectives of the study. After extensive revision, the final version of the English questionnaire was developed and translated to the local language that is Guragegna.

The ethical clearance was obtained from Wolkite University, College of Health Sciences Research Ethics Review Committee [15-17]. The survey was commenced after obtaining permission from Gurage Zonal Health Department and District Council. Informed verbal consent was obtained from each study subject. Each respondent was informed about the objective of the study and assurance of confidentiality.

\section{Method of analysis}

Binary logistic regression model was employed for the study because of the response variable (antenatal care service) is classified in to two categories that issues of maternal health services coded as 1 and not use is coded to 0 .The ratio of the success $(Y i=1)$ probability, $\mathrm{P}$

(xi) to that of $1-\mathrm{P}(x i)$ (failure $(\mathrm{Y}=0)$ probability) is given by: $\frac{P\left(\mathrm{x}_{i}\right)}{1-P\left(\mathrm{x}_{\mathrm{i}}\right)}$ is known as the odds of success. In terms of the odds, the logistic model can be written as $\frac{P\left(\mathrm{x}_{i}\right)}{1-P\left(\mathrm{x}_{\mathrm{i}}\right)}=\exp \left(\beta_{0}+\beta_{1} X_{i 1}+\beta_{2} X_{i 2}+\ldots+\beta_{p} X_{i p}\right) \quad$ and the odds in favour of success for multivariable logistic regression will be

$$
\left(\frac{P\left(\mathrm{x}_{i}\right)}{1-P\left(\mathrm{x}_{\mathrm{i}}\right)}\right)=\exp \left(\beta_{0}+\beta_{1} X_{i 1}+\beta_{2} X_{i 2}+\ldots+\beta_{p} X_{i p}\right), \quad i=1,2, \ldots, n .
$$

In which case, $P\left(\mathrm{x}_{i}\right)=\frac{e^{\beta_{0}+\beta_{1} X_{i 1}+\beta_{2} X_{i 2}+\ldots+\beta_{p} X_{i p}}}{1+e^{\beta_{0}+\beta_{1} X_{i 1}+\beta_{2} X_{i 2}+\ldots+\beta_{p} X_{i p}}}, i=1,2, \ldots, n$.

The parameter was interpreted by using Odds ratio. The odds ratio is the ratio between two odds. The odds of some event happening is defined as the ratio of the probability of occurrence to the probability of non-occurrence. That is, the odd of the event $\mathrm{E}$ is given by:

$$
\operatorname{odds}(E)=\frac{\operatorname{pr}(E)}{\operatorname{pr}(\text { not } E)}=\frac{\operatorname{pr}(E)}{1-\operatorname{pr}(E)} \text {. The odds ratio (OR) for two }
$$

events A and B given by: $O R=\frac{\operatorname{odd}(A)}{\operatorname{odd}(B)}$.

The maximum likelihood estimation method was used to estimate the parameters. In binary outcome each observation can be considered as an outcome of a Bernoulli trial, and hence for the $i^{\text {th }}$ observation: $P\left(Y=y_{i}\right)=p^{y_{i}}(1-p)^{1-y_{i}}$, assuming the $\mathrm{n}$ observations are independent, the likelihood function is $l(\beta)=\prod_{i=1}^{n} \mathrm{P}^{y_{i}}(1-\mathrm{P})^{1-y_{i}}=\prod_{i=1}^{n}\left(\frac{1}{1+e^{-x^{t} \beta}}\right)^{y_{i}}\left(\frac{e^{-x^{t} \beta}}{1+e^{-x^{t} \beta}}\right)^{1-y_{i}}$.

And the log likelihood function is given as:

$$
\begin{aligned}
& \log l(\beta)=\sum_{i=1}^{n} y_{i} \log \left(p_{i}\right)+\sum\left(1-y_{i}\right) \log \left(1-p_{i}\right), \\
& \Rightarrow \log l(\beta)=\sum_{i=1}^{n} y_{i} \log \left(\frac{1}{1+e^{-x^{t} \beta}}\right)+\sum_{i=1}^{n}\left(1-y_{i}\right) \log \left(\frac{e^{-x^{t} \beta}}{1+e^{-x^{\prime} \beta}}\right),
\end{aligned}
$$

It can be written as:

$$
L(\beta)=\sum_{i=1}^{n} y_{i} \log \left(\frac{1}{1+e^{-x^{t} \beta}}\right)+\sum_{i=1}^{n}\left(1-y_{i}\right) \log \left(\frac{e^{-x^{t} \beta}}{1+e^{-x^{t} \beta}}\right),
$$

where $L(\beta)=\log l(\beta)$

Hence by maximizing the above we can theoretically estimate the parameter $\beta$. To find an estimate of $\beta$ that maximizes $L(\beta)$, we differentiate $\mathrm{L}(\beta)$ with respect to each component of $\beta$ and set the resulting $(p+1)$ equations to zero.

\section{Results}

\section{Result of the descriptive analysis}

The distribution of mothers' use of antenatal care service with the corresponding determinants or explanatory variables is given in Table 1.

For age: From the table above the numbers of respondents whose age from $15-24$ is $54 \%$ were use antenatal care, $46 \%$ of them do not use antenatal care. From age 25-34, 25.2\% were use antenatal care, $74.8 \%$ of them do not use antenatal care. From age 35-44, 23.8\% were use antenatal care and $76.2 \%$ of them do not use antenatal care. And 
Citation: Alemayehu GM (2018) Statistical Analysis of Factors that Affect the Use of Maternal Health Care Services in Gubure Sub-City: SNNP, Ethiopia. J Biom Biostat 9: 399. doi: 10.4172/2155-6180.1000399

Page 3 of 6

\begin{tabular}{|c|c|c|c|c|}
\hline \multirow[t]{3}{*}{ Variable } & \multirow[t]{3}{*}{ Categories } & \multicolumn{2}{|c|}{ ANC } & \multirow[t]{3}{*}{ Total fr. } \\
\hline & & Use of ANC & Not use ANC & \\
\hline & & Fr. & Fr. & \\
\hline \multirow[t]{4}{*}{ Marital staus } & Marriage & $54 \%$ & $46 \%$ & $100 \%$ \\
\hline & Single & $25 \%$ & $75 \%$ & $100 \%$ \\
\hline & Divorced & $23 \%$ & $76 \%$ & $100 \%$ \\
\hline & Widowed & $17 \%$ & $83 \%$ & $100 \%$ \\
\hline \multirow[t]{3}{*}{ Child- number } & $<=4$ & $37 \%$ & $63 \%$ & $100 \%$ \\
\hline & 5 & $52.5 \%$ & $47.5 \%$ & $100 \%$ \\
\hline & $>5$ & $23 \%$ & $77 \%$ & $100 \%$ \\
\hline \multirow[t]{4}{*}{ Occupation } & House wife & $39 \%$ & $61 \%$ & $100 \%$ \\
\hline & Daily worker & $37.2 \%$ & $62.8 \%$ & $100 \%$ \\
\hline & Merchant & $21.4 \%$ & $78.6 \%$ & $100 \%$ \\
\hline & Other & $25.6 \%$ & $74.4 \%$ & $100 \%$ \\
\hline \multirow[t]{3}{*}{ Immunization } & Good & $38 \%$ & $62 \%$ & $100 \%$ \\
\hline & Medium & $48 \%$ & $52 \%$ & $100 \%$ \\
\hline & Bad & $23 \%$ & $77 \%$ & $100 \%$ \\
\hline \multirow[t]{2}{*}{ Health services } & No & $22.4 \%$ & $77.6 \%$ & $100 \%$ \\
\hline & Yes & $87.5 \%$ & $12.5 \%$ & $100 \%$ \\
\hline \multirow[t]{2}{*}{ Health information } & Yes & $56.7 \%$ & $43.3 \%$ & $100 \%$ \\
\hline & No & $24.3 \%$ & $75.7 \%$ & $100 \%$ \\
\hline \multirow[t]{4}{*}{ Age } & $15-24$ & $54 \%$ & $46 \%$ & $100 \%$ \\
\hline & $25-34$ & $25.2 \%$ & $74.8 \%$ & $100 \%$ \\
\hline & $35-44$ & $23.8 \%$ & $76.2 \%$ & $100 \%$ \\
\hline & $>44$ & $75 \%$ & $25 \%$ & $100 \%$ \\
\hline \multirow[t]{6}{*}{ Level of education } & Illiterate & $33.9 \%$ & $76.1 \%$ & $100 \%$ \\
\hline & Primary & $30.6 \%$ & $69.4 \%$ & $100 \%$ \\
\hline & Secondary & $22.7 \%$ & $77.3 \%$ & $100 \%$ \\
\hline & Certificate & $34.8 \%$ & $65.2 \%$ & $100 \%$ \\
\hline & Diploma & $67.1 \%$ & $32.9 \%$ & $100 \%$ \\
\hline & Degree other & $76 \%$ & $24 \%$ & $100 \%$ \\
\hline \multirow[t]{4}{*}{ Income } & $<500$ & $38.8 \%$ & $61.2 \%$ & $100 \%$ \\
\hline & $500-1000$ & $28.4 \%$ & $71.6 \%$ & $100 \%$ \\
\hline & $1000-1500$ & $38.7 \%$ & $61.3 \%$ & $100 \%$ \\
\hline & $1500-2000$ & $52.8 \%$ & $47.2 \%$ & $100 \%$ \\
\hline \multirow[t]{4}{*}{ Religion } & Orthodox & $31.7 \%$ & $68.3 \%$ & $100 \%$ \\
\hline & Muslim & $37.9 \%$ & $62.1 \%$ & $100 \%$ \\
\hline & Protestant & $39.5 \%$ & $50.5 \%$ & $100 \%$ \\
\hline & Other & $45.7 \%$ & $54.3 \%$ & $100 \%$ \\
\hline \multirow[t]{2}{*}{ Nutrition } & Balanced & $33.6 \%$ & $66.4 \%$ & $100 \%$ \\
\hline & Unbalanced & $76.8 \%$ & $22.2 \%$ & $100 \%$ \\
\hline
\end{tabular}

Table 1: Descriptive statistics of antenatal visit care on sampled data.

from age $\geq 45,75 \%$ were use antenatal care and $25 \%$ of them do not use antenatal care. Therefore as age increases women's are more user of maternal health services.

For marital status: According to our study the women from all respondents who are married are $54 \%$, single $25 \%$, divorced $23 \%$ and widowed $17 \%$ use antenatal care service respectively. From those who do use maternal health services are married $46 \%$, single $75 \%$, divorced $76 \%$ and widowed $83 \%$. Then from all marital status women's who are married are more users of maternal health services and divorced are more non user.

For child-number: Based on the result of the response of respondent's, women are who have child-number $<=4,37 \%,=5,52.5 \%$, $>5,23 \%$ use antenatal care services. Which indicates that women's who have small number of children more use antenatal care.

For occupation: When we summarize the answer of the respondents women's who were house wife $39 \%$, daily worker $37.2 \%$, merchant $21.4 \%$ and others $25.6 \%$ use antenatal care service. and from those respondents the non user of maternal health services are house wife $61 \%$, daily worker $62.8 \%$, merchants $78.6 \%$ and others $74.4 \%$ respectively.

For health services: We describe that womens who get heath service $22.4 \%$ use antenatal care. and who do not get health service $87.5 \%$ use antenatal care. and from them women's who say there is adequate health services users are $77.6 \%$ and no are $12.5 \%$ do not use antenatal care.

For education level: We describe that women's who are illiterate $33.9 \%$, primary $30.6 \%$, secondary $22.7 \%$, certificate $34.8 \%$, diploma $67.1 \%$ and degree $\&$ above $76 \%$ use antenatal care. From those women's who are non user of antenatal care are illiterate $76.1 \%$, primary $69.4 \%$, secondary $77.3 \%$, certificate $65.2 \%$, diploma $32.9 \%$ and degree $\&$ above $24 \%$ respectively.

For income: - from the above table the numbers of women whose 
income level are $<500,38.8 \%, 500-100038.7 \%, 1000-150052.8 \%$ and $1500-20008(5.5 \%)$ are use of antenatal care and from over responses on income who are the no user are $<50061.2 \%$ ), 500-1000 71.6\%, 1000$150061.3 \%$ and $1500-200047.2 \%$ respectively.

For religion: When we examine or summarize the answer of the respondents women's whose answer are orthodox 31.7\%, Muslim $37.9 \%$, protestant $39.5 \%$ and other $45.7 \%$ use antenatal care. and from those respondents the non user of maternal health services are orthodox $68.3 \%$, Muslim $62.1 \%$, protestant $50.5 \%$ and other $54.3 \%$ respectively. Based on those values the women's whose religion is others are the more user of maternal health services and orthodox are the smaller user.

For nutrition: From the above table the numbers of women whose nutrition are balanced $33.6 \%$ and unbalanced $76.8 \%$ use antenatal care service. And $66.4 \%$ of women who get balanced diet are non user of antenatal care.of those women who did not get balanced diet $22.2 \%$ are do not use antenatal care service.

Hosmer and Lemeshow test was used to test goodness of fit of the modelthen the value of the Hosmer- Lemeshow goodness-of-fit test is statistical significance which value is greater than 0.05 (i.e. $0.211>0.05$ ), we fail to reject the null hypothesis that there is no difference between observed data and model-predicted values, implying that the model fits the data at an acceptable level, this proves that the predicted data are not significantly different from the observed data. There for the model fits data in well.

\section{Determinants of antenatal care visit service}

The output of binary logistic regression indicates that marital status $(\mathrm{p}<0.001)$, occupation $(\mathrm{p}<0.014)$, immunization $(\mathrm{p}<0.006)$, health services $(p<0.032)$, health information $(p<0.009)$ were statistically significant and the output of the binary logistic regression outcome is showed in Table 2.

Interpretation about the factors of ANC was done from the above table based on the coefficients of each variable.

For marital status $=\beta 1$, from the parameter estimation above; the chi-square statistics (Wald) $=15.774$ is greater than $\mathrm{X} 20.05,1=7.81$ the $p$-values for marital status $(\beta 1)=0.001$ is less than 0.05 level of significance. Thus based on this result we see that the coefficient of marital status is significant to the model. The odd ratio for married is 201. 754 which indicates that married mothers are 201.754 more likely to use antenatal care as compared to widowed mothers (the reference group) controlling for other variables in the model. Single mothers are $(\mathrm{OR}=0.024) 0.024$ times less likely to use maternal health care as compared to widowed mother. The odds ratio $(\mathrm{OR}=0.357)$ shows that divorced mothers are 0.357 times less likely to use antenatal health care.

For occupation $=\beta 2$, from the parameter estimation above; the chi-square statistics (Wald) $=10.644$ is greater than X20.05, $1=7.81$ the $\mathrm{p}$-values for occupation $\left(\beta_{2}\right)=0.014$ is less than 0.05 level of significance. Thus based on this result we see that the coefficient of occupation is significant to the model.

The variable occupation, the reference category is other, mothers who are housewife are $0.009 \%$ less likely to use antenatal care as compared to those who are other in controlling for other variables in the model. Daily worker mothers are 2.839 times more likely to use antenatal health care as compared to the reference category (other). the odds ratio $\mathrm{OR}=4.722$ indicates that merchant mothers are 4.722 times more likely use antenatal health care as compared the reference categories by controlling other variables in the model.

For immunization $=\beta 3$ from the parameter estimation above; the chi-square statistics $($ Wald $)=10.284$ is greater than X20.05, $1=5.991$ the p-values for immunization $\left(\beta_{3}\right)=0.006$ is less than 0.05 level of significance. Thus based on this result we see that the coefficient immunization is significant to the model.

For the variable immunization, the reference category is bad, the odds of mothers being get antenatal care $286.310 \%$ higher for mother that say immunization is goo than who say immunization is bad by controlling other variables in the model.

For health information $=\beta 5$, from the parameter estimation above; the chi-square statistics (Wald) $=6.729$ is greater than $\mathrm{X} 20.05,1=3.84$ the $p$-values for health information $(\beta 6)=0.009$ is less than 0.05 level of significance. Thus based on this result we see that the coefficient health information is significant to the model.

For health services $=\beta 4$, from the parameter estimation above; the chi-square statistic (Wald) $=4.600$ is greater than $\mathrm{X} 20.05,1=3.84$ the $p$-values for health services $(\beta 4)=0.032$ is less than 0.05 level of significance. Thus based on this result we see that the coefficient health services is significant to the model.

Lastly for the variable health services the reference category is yes, mothers who do not get health service are $0.008 \%$ less likely to use

\begin{tabular}{|c|c|c|c|c|c|c|c|c|}
\hline \multirow[t]{2}{*}{ Variables } & \multirow[t]{2}{*}{ B } & \multirow[t]{2}{*}{ S.E } & \multirow[t]{2}{*}{ Wald } & \multirow[t]{2}{*}{ Df } & \multirow[t]{2}{*}{ Sig. } & \multirow[t]{2}{*}{$\operatorname{Exp}(B)$} & \multicolumn{2}{|c|}{$95 \%$ C.I for $\exp (B)$} \\
\hline & & & & & & & Lower & Upper \\
\hline Marital stat & & & 15.774 & 3 & .001 & & & \\
\hline Marital stat (1) & 5.307 & 1.846 & 8.268 & 1 & .004 & 201.754 & 5.412 & 7521.667 \\
\hline Marital stat (2) & -3.729 & 1.798 & 4.303 & 1 & .038 & .024 & .001 & .814 \\
\hline Martila stat (3) Ref (widowed) & -1.029 & 1.175 & .767 & 1 & .381 & .357 & .036 & 3.576 \\
\hline Occupation & & & 10.644 & 3 & .014 & & & \\
\hline Occupation (1) & -4.667 & 1.810 & 6.651 & 1 & .010 & .009 & .000 & .326 \\
\hline Occupation (2) & 1.043 & 1.245 & .702 & 1 & .402 & 2.839 & .247 & 32.573 \\
\hline Occupation (3) Ref (other) & 1.552 & 1.408 & 1.215 & 1 & .270 & 4.722 & .299 & 74.587 \\
\hline Immunization & & & 10.284 & 2 & .006 & & & \\
\hline Immunization (1) & 5.657 & 2.022 & 7.831 & 1 & .005 & 286.310 & 5.446 & 15053.253 \\
\hline Immunization (2) Ref (bad) & 3.558 & 1.801 & 3.902 & 1 & .048 & 35.103 & 1.028 & 1198.651 \\
\hline Health service (1) Ref (yes) & -4.829 & 2.252 & 4.600 & 1 & .032 & .008 & .000 & .660 \\
\hline Health inform (1) Ref (no) & 3.901 & 1.504 & 6.729 & 1 & .009 & 49.463 & 2.595 & 942.869 \\
\hline Constant & -9.379 & 5.102 & 3.379 & 1 & .066 & .000 & & \\
\hline
\end{tabular}

Table 2: The estimate of covariates for the use of maternal health care service in the final model. 
antenatal care as compared those who get health service controlling other variables in the model.

\section{Discussion}

The study was attempted to identify some determinant of the use of maternal health services, in this research there were 1624 total populations and the behaviour of this total population was estimated based on the sample size 145. According to our study the result of the descriptive analysis showed that the number of women's those who are the user of maternal health services are very less as well as many of them have no essential information about maternal health services and who have information are does not the user because of there is no adequate health services or public services which gives essential services for mothers immunization but in the study of Abera there is adequate health services. Generally when we compare present findings with previous results listed in the literature that of the study, findings from the previous study show clearly the central role that relationships and patients' expectations play about dependence of using maternal health services is on: - Distribution of women and girls, Place of births birth is occurred, Professions of health care, health facility and etc. Current study (our study) clearly showed that the dimension of using maternal health services includes: occupation, education level, age, income of household, and etc. From those dimensions the odds of complete immunization status among women's who have medium knowledge and no on benefit of maternal service are slightly less than those with have knowledge on it by using odd ratio for women who have medium knowledge $\mathrm{OR}=31.1 \%$ less, have no $\mathrm{OR}=2.8 \%$ less than who have knowledge and women's who have whose occupation are daily worker are highly than those with occupation are housewife and whose occupation are merchant, other are slightly less than those with occupation whose occupation are house wife. Women's whose occupation are daily worker are $\mathrm{OR}=9 \%$ less, merchant $\mathrm{OR}=83.9 \%$ more, other $\mathrm{OR}=72.2 \%$ more, are than whose occupation are housewife. But the result is also consists with Abera which reports that, occupation of women's odds ratio of merchant is increased $1.54 \%$ and occupation women who have is other has decreased by a factor of 0.183 . Similarly, the odds of women who have medium knowledge regarding to immunization are as we have seen in the table the odds ratio is decreased by $6.5 \%$ and no are increased by $\mathrm{OR}=1.21 \%$. But according to Gurmessa, women who have occupation are daily worker are more likely to be fully immunized than women's whose occupation are house wife.

For the variable have no knowledge (information) of maternal health services are $59.5 \%$ less likely to as compared to those who have controlling for other variables in the model. The study contradicts with Gurmessa which report that opposite of our conclusion.

\section{Conclusion}

Since the aim of maternal health service is to improve the health of mothers it would be essential to study the factors that can improve the performance of the service. The health community is largely dependent upon their living conditions and life styles. The factors which significantly influence health status of women are physical, social and economic environments.

From the result of our study there is association between existence of adequate health services and maternal health services. We then conclude that the more if there is an adequate health service then every woman are the user of maternal health services because of there is adequate health services around of them. There is association between child-number of woman and maternal health services. When we say this if women's have more children than they are know the advantage of maternal health services because of they are used repeatedly as soon as they get child. And also there is association between marital status and maternal health services. When women's are married they are the user and if they have husband they get pregnancy.

Similarly, immunization of women has an effect on maternal health services. That is, there is association between immunization of women and utilization of maternal health services. There is association between women's knowledge regarding to maternal health services (health information) and maternal health services. More they have positive information about the advantage of maternal health services they are more the user.

The result of study also indicated that there is no association between nutrition and maternal health services and also there is no association between income of house hold of women's and maternal health service.

\section{Acknowledgement}

We the researcher would like to thank the office of Mothers and children of Gubure sub-city that helped us during data collection by giving appropriate data as well as the population of Gubure sub-city for their willingness to give appropriate answer for the questions.

\section{Funding}

We did not receive external funding for this research.

\section{Authors' Contributions}

Gedif Mulat designed the study, performed the statistical analysis, prepared the manuscript Gedif M. collected the data and interpreted the results. The authors also read and approved the final manuscript.

\section{Author's Information}

The author currently works at Wolkite University, Wolkite, Ethiopia. He serve as a Lecturer and Researcher in Biostatistics Department, Institute of Public Health. Gedif obtained both Master of Science in Biostatistics and Bachelor of Science in Statistics from University of Gondar, Ethiopia. Tariku obtained his Bachelor degree at Wolkite University.

\section{Competing Interests}

The author declares that he has no competing interests.

\section{Consent for Publication}

This manuscript does not contain any individual person's data.

\section{Ethics Approval and Consent to Participate}

The ethical clearance was obtained from Wolkite University, College of Health Sciences Research Ethics Review Committee. The survey was commenced after obtaining permission from Gurage Zonal Health Department and District Council. Informed verbal consent was obtained from each study subject. Each respondent was informed about the objective of the study and assurance of confidentiality.

\section{References}

1. Alden KR, Lowdermilk DL, Cashion MC, Perry SE (2013) Maternity and Women's Health Care-E-Book. Elsevier Health Sciences.

2. Cheng CY, Fowles ER, Walker LO (2006) Postpartum maternal health care in the United States: A critical review. J Perinat Educ 15: 34-42.

3. Okoth CA (2014) Utilization Of Skilled Birth Attendants Among Women Of Reproductive Age In Central Division, Kajiado County, Kenya(Doctora Dissertation, School Of Public Health, Kenyatta University).

4. World Health Organization (2005) The World Health Report 2005: Make every mother and child count. World Health Organization.

5. Tarekegn SM, Lieberman LS, Giedraitis V (2014) Determinants of maternal health service utilization in Ethiopia: analysis of the 2011 Ethiopian Demographic and Health Survey. BMC Pregnancy Childbirth 14: 161. 
Citation: Alemayehu GM (2018) Statistical Analysis of Factors that Affect the Use of Maternal Health Care Services in Gubure Sub-City: SNNP, Ethiopia. J Biom Biostat 9: 399. doi: 10.4172/2155-6180.1000399

6. Worku AG, Yalew AW, Afework MF (2013) Factors affecting utilization of skilled maternal care in Northwest Ethiopia: a multilevel analysis. BMC Int Health Hum Rights 13: 20.

7. Sibanda A, Woubalem Z, Hogan DP, Lindstrom DP (2003) The Proximate Determinants of the Decline to Below-replacement Fertility in Addis Ababa, Ethiopia. Stud Fam Plann 34: 1-7.

8. Central Statistical Agency (CSA): January 2007 Trends in demographic and reproductive health indicators in Ethiopia Addis Ababa.

9. Koblinsky M, Tain F, Gaym A, Karim A, Carnell M, et al. (2010) Responding to the maternal health care challenge: The Ethiopian Health Extension Program. Ethiop J Health Dev 24: 105-109.

10. Ethiopia demographic and health survey (EDHS: 2005) maternal care seeking Behavior in Ethiopia: Ethiopian society of population studies.

11. World Health Organization, 2015. World report on ageing and health. World Health Organization.
12. Hiluf M, Fantahun M (2008) Birth preparedness and complication readiness among women in Adigrat town, north Ethiopia. Ethiop J Health Dev 22: 14-20.

13. Babalola S, Fatusi A (2009) Determinants of use of maternal health services in Nigeria-looking beyond individual and household factors. BMC Pregnancy Childbirth 9: 43 .

14. Ross JA, Campbell OM, Bulatao $R$ (2001) The maternal and neonatal programme effort index (MNPI). Trop Med Int Health 6: 787-798.

15. Furuta M, Mori R (2008) Factors affecting women's health-related behaviors and safe motherhood: a qualitative study from a refugee camp in eastern Sudan. Health Care Women Int 29: 884-905.

16. Casella G, Berger RL (2002) Statistical inference (Vol. 2) Pacific Grove, CA: Duxbury.

17. Klassen JA, Morrison ML, Mathewson HA, Rosenthal GG, Wilkins RN (2012) Canopy characteristics affect reproductive success of golden-cheeked warblers. Wildlife Society Bulletin 36: 54-60. 